\title{
A New Method of Calculation of the Exact Weight of Wet Aggregates and Adjusted Weight of Water in Dosing and Production of Concrete
}

\author{
Milan KEKANOVIĆ
}

\begin{abstract}
Author of this paper presents a new method of determining the exact corrected mass of wet aggregates and water in the making and production of concrete. Namely, in the existing process [4] for determining the mass of wet aggregates which are dosed into the mixer, there is an overabundance measure of water and shortage of aggregates in the mixture. The error increases with the increase of the humidity of aggregate. Excess water in the mixture adversely affects the strength, resistance and durability of concrete. The author gives a new functional dependence (15) for calculating the wet aggregate mass $\left(m^{* *}{ }_{o a}\right)$ in iterative procedure with pre-set accuracy defined by the formula (14). The author also provides a second formula (16) for direct calculation of the wet aggregate mass $\left(m^{* *}{ }_{o a}\right)$ that is exact. The water mass $\left(m^{* *}{ }_{w}\right)$ is easily calculated using the formula (17), where the wet aggregate mass is previously calculated. This method of calculating the exact mass of the liquid and the exact mass of the solid components (mass of the material) according to the presented procedure and the new formulas (15), (16) and (17) is applicable to all types of mixtures that are used in the construction, food and pharmaceutical industries.
\end{abstract}

Keywords: aggregate; concrete; mixtures; water; weight; wet

\section{INTRODUCTION}

In factories, concrete production is carried out according to precise recipes by weighing the mass of each component: cement mass $\left(m_{c}\right)$, the mass of fully dry aggregates $\left(m_{o a}\right)$, mass of reduced water $\left(m^{*}{ }_{w}\right)$ and the mass of additives $\left(m_{a d}\right)$. However, the aggregates are usually moist, hence there is a real problem in measuring masses of dry aggregate and water. By measuring the mass of a certain volume of aggregate, we indirectly measure the mass of water present in the aggregate. If the humidity of the aggregate is known, we are able to calculate the mass of water in the aggregate. The current methodology [4] of calculating the amount of water present in the aggregate is not sufficiently precise. This means, as of today, that neither the mass of water, nor the mass of aggregate calculated and dosed into the mixer during the production of concrete, is sufficiently accurate and precise. As the humidity of aggregate increases, the error in calculating also increases. The new method of determining the exact masses of the aggregates and water in the production of concrete, presented in this scientific article, is created as a result of detailed observation of the physical process of wet aggregate dosing, which shows that the increased mass of aggregates cannot be calculated by simply multiplying the free moisture in the aggregate $H_{a}(\%)$ with the mass of aggregate $\left(m_{o a}\right)$.

\section{THE CURRENT WAY OF APPROXIMATE AND INSUFFICIENTLY PRECISE CALCULATION OF THE MASSES OF THE WATER AND AGGREGATES IN THE PROCESS OF WET AGGREGATE DOSING}

As mentioned in the introductory part, simply multiplying the mass of dry aggregate $\left(m_{o a}\right)$ with the humidity of the aggregate $H_{a}(\%)$, does not give us sufficiently accurate results. The mass of aggregate, which is calculated as the difference between the mass of completely dry and the mass of aggregate increased by the percentage of humidity, also contains a certain water mass, because the aggregate is moist. The mass of water contained in the corrected aggregate mass represents the size of the error, because the mass of dry aggregate has not been reached (according to the projected mixture of fresh concrete). This means that the mass of dry aggregate, according to the recipe for fresh concrete, will be reduced by the mass of water contained in the corrected mass of the aggregate, while the mass of water will be increased in relation to the recalculated mass of water in the recipe.

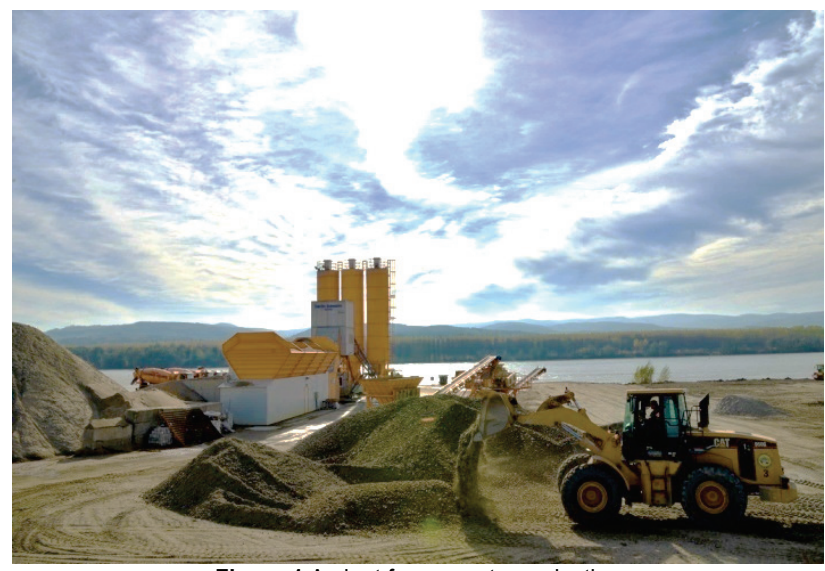

Figure $1 \mathrm{~A}$ plant for concrete production

The humidity of the aggregate is calculated as:

$H_{a}=\frac{m_{o w}-m_{o a}}{m_{o a}} \cdot 100, \%$

where: $H_{a}$ - The humidity of a particular fraction of the aggregate $(\%) ; m_{o w}$ - The moist aggregate mass $(\mathrm{kg}) ; m_{o a}$ The mass of the completely dry aggregate, calculated according to the recipe of the fresh concrete $(\mathrm{kg})$

In the present methods for calculating the real, the increased aggregate mass, calculation is done through the following equation:

$m_{o a}^{*}=m_{o a} \cdot\left(1+\frac{H_{a}}{100}\right), \mathrm{kg}$

or, otherwise: 
$m_{o a}^{*}=\left(m_{o a 1}+\Delta m_{a w 1}\right)+\Delta m_{o a 1}, \mathrm{~kg}$

where: $m_{o a 1}$ - A part of the mass of completely dry aggregate contained in the total mass of the moist aggregate $(\mathrm{kg}) ; \Delta m_{a w 1}$ - A part of the mass of water in the total mass of the moist aggregate $(\mathrm{kg}) ; \Delta m_{o a 1}$ - The additional mass of the (moist) aggregate, which is the result of the humidity of the aggregate $H_{a}(\mathrm{~kg})$.

If there is no humidity $\left(H_{a}=0\right)$, then there is no water in the aggregate $\left(\Delta m_{a w 1}=0\right)$, so there is no need to put in the additional aggregate $\left(\Delta m_{o a 1}=0\right)$.

Developed form of the Eq. (3) is:

$m_{o a}^{*}=\left(m_{o a 1}+\Delta m_{a w 1}\right)+\left(m_{o a 2}+\Delta m_{a w 2}\right), \mathrm{kg}$

where: $m_{o a 2}$ - A part of the dry aggregate mass in the additional mass of the moist aggregate $\Delta m_{o a 2}(\mathrm{~kg}) ; \Delta m_{a w 2}$ A part of the water mass in the additional mass of the moist aggregate $\Delta m_{o a 2}(\mathrm{~kg})$.

Mass $\Delta m_{a w 2}$ represents an error, because it is not covered by the mass of water in the process of dosing the aggregate mass. This means that, in the concrete production, the mass of required aggregates in the dry state will be reduced by the mass - $\Delta m_{a w 2}$. Likewise, the water mass in the concrete will be increased by the same mass $\Delta m_{a w 2}$.

For a better understanding of the physical state of water in a moist aggregate used in concrete production, the Eq. (2) can be written in a developed form:

$$
\begin{aligned}
& m_{o a}^{*}=\left[\left(m_{o a}-m_{o a} \frac{H_{a}}{100}\right)+m_{o a} \frac{H_{a}}{100}+\right. \\
& \left.+m_{o a}\left(\frac{H_{a}}{100}-\frac{H_{a}}{100} \cdot \frac{H_{a}}{100}\right)+m_{o a} \frac{H_{a}}{100} \cdot \frac{H_{a}}{100}\right]
\end{aligned}
$$

or, otherwise:

$$
\begin{aligned}
& m_{o a}^{*}=\left\{\left(m_{o a}-m_{o a} \frac{H_{a}}{100}\right)+m_{o a} \frac{H_{a}}{100}+\right. \\
& \left.+m_{o a}\left[\frac{H_{a}}{100}-\left(\frac{H_{a}}{100}\right)^{2}\right]+m_{o a}\left(\frac{H_{a}}{100}\right)^{2}\right\}
\end{aligned}
$$

which brings us to the starting Eq. (2):

$$
m^{*}{ }_{o a}=m_{o a} \cdot\left(1+\frac{H_{a}}{100}\right), \mathrm{kg}
$$

The mass $\left(m^{*}\right.$ oa $)$ is the aggregate mass increased by the water mass which is present in the aggregate $\Delta m_{a w 1}$ but the result yielded in this fashion is not sufficiently precise, because the mass which increases the mass of wet aggregate $\Delta m_{o a 1}=\Delta m_{a w 1}=\left(m_{o a 2}+m_{a w 2}\right)$ consists of humidity $H_{a}(\%)$, too, which means that there is water mass $\Delta m_{a w 2}$, as can be seen from the Eq. (4). The current method of calculating the mass of wet aggregate neglects the presence of water $\Delta m_{a w 2}$ in the corrected aggregate mass $m^{*}$ oa.
The mass of aggregate which is increased by $\Delta m_{a w 1}$ is calculated in a manner that follows:

$$
\begin{aligned}
& \Delta m_{a w 1}=m_{o a} \cdot\left(\frac{H_{a}}{100}\right), \mathrm{kg} \\
& \Delta m_{o a 1}=\Delta m_{a w 1}=m_{o a 2}+\Delta m_{a w 2}, \mathrm{~kg}
\end{aligned}
$$

Based on the presented analysis, we conclude that the aggregate mass $\left(m^{*}\right.$ oa $)$ determined by the existing methods would have to be reduced with the value of the water mass - $\Delta m_{\text {oa } 2}$, which is located in the increased aggregate mass $\Delta m_{\text {oa } 1}$, compared to the present aggregate humidity $H_{a}(\%)$. This water mass is calculated according to the formula (8), so we get:

$$
\begin{aligned}
& \Delta m_{a w 2}=\Delta m_{o a 1} \cdot \frac{H_{a}}{100}= \\
& =m_{o a} \cdot\left(\frac{H_{a}}{100}\right) \cdot \frac{H_{a}}{100}=m_{o a} \cdot\left(\frac{H_{a}}{100}\right)^{2}, \mathrm{~kg}
\end{aligned}
$$

Using the existing methods, the mass of reduced water $\left(m^{*}{ }_{w}\right)$ is calculated by subtractions of the mass of projected water in the concrete $-m_{w}$, from the mass of water present in the aggregate:

$$
m_{w}^{*}=m_{w}-\Delta m_{a w 1}=m_{w}-\left(m_{o a}^{*}-m_{o a}\right), \mathrm{kg}
$$

The water mass $\left(m^{*}{ }_{w}\right)$ was reduced only by the water mass present in the aggregate - $\Delta m_{a w 1}$, rendering the result not sufficiently precise. In fact, there is an error in calculating the exact amount of water present in the aggregate, because it does not include the water mass $\Delta m_{a w 2}$ (see Eq. (4)). The water mass in the fresh concrete will be increased by the water mass - $\Delta m_{a w 2}$, in relation to the projected water as originally designed in the recipe.

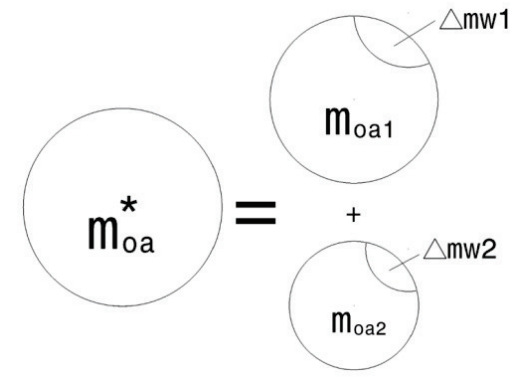

Figure 2 Graphic presentation of the present procedure for measuring the moist aggregate mass $\left(m^{*}{ }_{o a}\right)$ which is consisted of the dry aggregates $\Delta m_{o a 1}$ and the water mass in aggregate $\Delta m_{a w 1}$, so it is necessary to increase aggregate mass with the size of the water mass present in the aggregate $\Delta m_{a w 1}=m_{o a 2}+m_{a w 2}$, where the value of $\Delta m_{a w 2}$ represents a fault in this process, because such process ignores that the corrected addition to the aggregate is wet (ignores the present value of the water mass $\Delta m_{a w 2}$ )

The current method of calculating the mass of aggregate and water during the dosing into a concrete mixer would be correct if two conditions are met, and only then. The first condition is that $\Delta m_{a w 1}=0$, which means that the aggregate humidity is equal to 0 . This practically means that the aggregate must be dry at the time of dosing into the mixer which is not feasible. The second condition is that $\Delta m_{a w 3}=0$, which means that the aggregate mass 
which is added in the mass $\Delta m_{o a 1}$, which is also fed into the mixer, would have to be completely dry, too.

This leads us to the conclusion that the current method of calculating the aggregate mass and the water mass in the concrete production is not sufficiently accurate and precise. The error in this method increases as the aggregate humidity $H_{a}(\%)$ increases. If the aggregate humidity is little, then the error is smaller.

\section{A NEW METHOD OF DESIGNING THE ACCURATE AGGREGATE AND WATER MASSES IN THE CONCRETE PRODUCTION}

The purpose of designing a mixture of fresh concrete and determining the cement, aggregate, water and additive masses is production of concrete mixtures, which has its pre-determined quality (class) $\mathrm{C} f_{c k} / f_{c k \text {,cube }}$.

The individual aggregate fractions have different humidity $H_{a}(\%)$ levels and bulk densities. Determining the mass of individual fractions is done by multiplying the total volume of aggregates $\left(V_{a}\right)$ with granulometric participation of individual fractions $\left(\Delta_{p i}\right)$ and its bulk density $-\gamma_{z, a i}$ :

$$
V_{a i}=V_{a} \cdot \Delta_{p i} \cdot \gamma_{z, a i}, \mathrm{~m}^{3}
$$

In order to provide a clearer and simpler showing of the problem of calculating the wet aggregate and water masses in the concrete production, the author is analysing one type of aggregate and one fraction which have a single (the same) humidity $H_{a}(\%)$.

In the process of concrete production, it is necessary to take into account the humidity of each fraction of the aggregate, especially the first fraction with the smallest grains $(0 / 4 \mathrm{~mm})$. As the aggregate grain is smaller, its specific surface $\left(S=\mathrm{cm}^{2} / \mathrm{g}\right)$ is greater. Accordingly, the humidity of the fine granules $H_{a}(\%)$ may be high, because those granules have a large specific surface area $(S=$ $\left.\mathrm{cm}^{2} / \mathrm{g}\right)$. Similarly, the water absorption $u(\%)$ is higher for a smaller grain, because absorption is uniform at the surface and in the central part of the particles of such aggregate, thanks to the fact that the route is shorter and the resistance to the absorption is smaller.

Humidity $H_{a}(\%)$ of the first fraction can be $10 \%$ (or even more than $20 \%$ ). The mass of water placed around the aggregate grains must be accurately calculated and subtracted from the mass of water which was calculated as required in the concrete production.

The wet aggregate mass must be increased because the calculated mass of aggregate, which is fed into the mixer in the process of concrete production because this means that the aggregate is completely dry. In the attempt to explain the physical aspect of the problem, calculations of the actual mass of aggregate that needs to be dosed in the concrete production will be conducted iteratively. At the end, we will give a new equation of this, an iterative, process, and a new equation for a direct calculation of the aggregate and water mass without iteration.

The iterative procedure is performed until the value of the additional aggregate mass $\Delta m_{o a 1}$ is approximately equal to zero, because then the water mass $\Delta m_{a w 1}$ in the aggregate will be equal to zero, which is necessary to avoid dosing errors during the implementation of the dry aggregate.
Similarly, we are avoiding errors that occur due to the increased water mass.

$$
\begin{aligned}
& m^{* *}{ }_{o a}=m_{o a} \cdot\left(1+\frac{H_{a}}{100}\right)+m_{o a} \cdot \frac{H_{a}}{100} \cdot \frac{H_{a}}{100}+ \\
& +m_{\text {oa }} \cdot \frac{H_{a}}{100} \cdot \frac{H_{a}}{100} \cdot \frac{H_{a}}{100}+m_{o a} \cdot \frac{H_{a}}{100} \cdot \frac{H_{a}}{100} \cdot \frac{H_{a}}{100} \cdot \frac{H_{a}}{100}+\cdots, \mathrm{kg}
\end{aligned}
$$

or, otherwise:

$$
\begin{aligned}
& m_{o a}^{* *}=m_{o a} \cdot\left(1+\frac{H_{a}}{100}\right)+m_{o a} \cdot\left(\frac{H_{a}}{100}\right)^{2}+m_{o a} \cdot\left(\frac{H_{a}}{100}\right)^{3}+ \\
& +m_{o a} \cdot\left(\frac{H_{a}}{100}\right)^{4}+\cdots+m_{o a} \cdot\left(\frac{H_{a}}{100}\right)^{n-1}, \mathrm{~kg}
\end{aligned}
$$

where: $m_{o a}^{* *}$ - The increased aggregate mass that is exact (increased by the mass of water present in the aggregate, because the aggregate is moist with a moisture level $H_{a}$ $(\%)) ; m_{o a}$ - The mass of the completely dry aggregate calculated in the concrete recipe for the required class of concrete $\mathrm{C} f_{c k} / f_{c k \text {, cube. }}$.

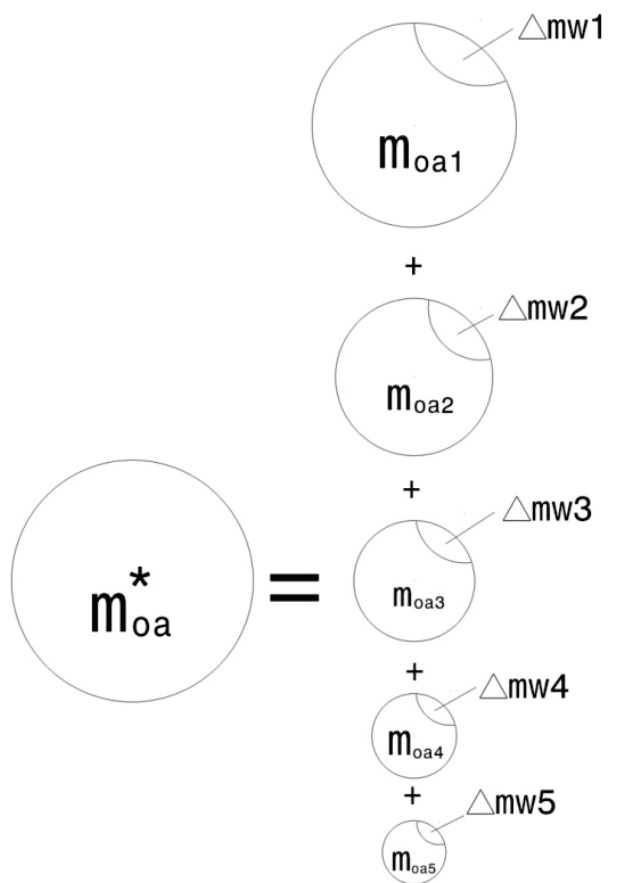

Figure 3 Graphic representation of iterative procedure for dosing the mass of the aggregate of certain humidity to the mixer until the mass $\Delta m_{o a 5}$ has a negligibly small value which means that the mass of water in this small addition of aggregate is $\Delta m_{o a 5} \approx 0$

This illustration clearly shows that the second constituent of the equation again contains water with its added aggregate mass, which means that for that mass of water, the mass of aggregate fractions is reduced.

The author provides an iterative method for dosing the aggregate and water as a contribution to the technology of concrete production. This leads to a result which yields a low error in aggregate dosing which strives towards zero. The number of iterative steps is determined for a pre-set accuracy $\left(l_{n} q\right)$ : 
$k \leq \frac{l_{n} q}{l_{n} H_{a}}$

where: $k$ - The number of addends for a given accuracy $q$ ( $k$ is adopted as an integer); $l_{n} q$ - The required accuracy (for accuracy to the fifth decimal place $q=0.00001) ; m_{o a}$ - The aggregate mass in a completely dry state to be dosed into the mixer according to the recipe for concrete production $(\mathrm{kg}) ; l_{n} H_{a}$ - Logarithm of a number that represents the aggregate humidity $H_{a} ; H_{a}$ - Humidity of the individual aggregate fraction. form:

The method described above is reduced to the general

$m_{o a}^{* *}=\sum_{n=1}^{k} m_{o a} \cdot H_{a}^{n-1} ; k \leq \frac{l_{n} q}{l_{n} H_{a}}, \mathrm{~kg}$

where: $m_{o a}^{* *}$ - The increased, exact aggregate mass with current humidity which should be dosed into the concrete (The aggregate mass is increased by the water mass present in the aggregate).

The aggregate mass $m^{* *}{ }_{o a}$ is calculated with the required accuracy $(k)$.

The author gives a direct method for calculating the aggregate mass $-m^{* *}{ }_{o a}$ :

$$
m_{o a}^{* *}=m_{o a} \cdot \frac{1}{\left(1-\frac{H_{a}}{100}\right)}, \mathrm{kg}
$$

The same or similar result is obtained by the direct method using the Eqs. (16) and (15) with the use of the iterative procedure. The identity of the results acquired by the Eqs. (16) and (15) depends on the required accuracy $(q),\left(q=1 / 10^{n}\right), 2<n<10$.

For practical reasons, we recommend direct method (16).

After determining the mass of wet aggregates, access to the calculation of the mass of water:

$$
m^{* *}=m_{w}-\left(m_{o a}^{* *}-m_{o a}\right), \mathrm{kg}
$$

where: $m^{* *}{ }_{w}$ - The reduced, exact water mass which is to be dosed into the mixer in the production of fresh concrete mixtures; $m_{w}$ - The water mass which is calculated in the recipe for the required concrete class under the assumption that the aggregate is completely dry.

\subsection{Comparative Analysis of the Results Obtained in Three Manners}

A practical example of the calculation for concrete production, if the aggregate mass in dry condition and water mass are calculated to be $m_{o a}=1935 \mathrm{~kg}$ and $m_{w}=$ $174 \mathrm{~kg}$, respectively, is analyzed. The aggregate moisture in the phase of concrete production and dosing in the mixer is $H_{a}=7,9 \%$.

\begin{tabular}{|c|c|c|c|}
\hline Mass/Method & $\begin{array}{l}\text { The Current method for calculating } \\
\text { (using Eq. (2) and Eq. (10)) } \\
\qquad m_{o a}^{*}=m_{o a} \cdot\left(1+\frac{H_{a}}{100}\right)\end{array}$ & $\begin{array}{c}\text { A New iterative method } \\
\text { (using Eq. (15) and Eq. (17)) } \\
m_{o a}^{* *}=\sum_{n=1}^{k} m_{o a} \cdot H_{a}^{n-1} ; k \leq \frac{l_{n} q}{l_{n} H_{a}}\end{array}$ & $\begin{array}{l}\text { A New direct method } \\
\text { (using Eq. (16) and Eq. (17)) } \\
m_{o a}^{* *}=m_{o a} \cdot \frac{1}{\left(1-\frac{H_{a}}{100}\right)}\end{array}$ \\
\hline$m_{o a}^{*}$ & $2087,865 \mathrm{~kg}$ & - & - \\
\hline$m^{* *}{ }_{o a}$ & - & $2100,961 \mathrm{~kg}$ & $2100,977 \mathrm{~kg}$ \\
\hline$m_{w}^{*}$ & $21,135 \mathrm{~kg}$ & - & - \\
\hline$m_{w}^{* *}$ & - & $8,039 \mathrm{~kg}$ & $8,023 \mathrm{~kg}$ \\
\hline
\end{tabular}

Table 1 Results obtained by the current method and methods presented above

where: $m^{*}{ }_{o a}$ - The increased wet aggregate mass to be dosed into the mixer using the current method; $m^{* *}$ oa - The increased, exact wet aggregate mass to be dosed into the mixer using new methods presented above; $m^{*}{ }_{w}$ - The reduced water mass to be dosed into the mixer using today's approximate procedure; $m^{* *}{ }_{w}$ - The reduced water mass using new, exact procedure that needs to be conveyed in the concrete production (reduced by the quantity of water contained in the aggregate).

There are differences in the masses of aggregate and water at the point where the aggregate humidity is $H_{a}=$ $7,9 \%$. In particular, the presence of water is increased mostly in the concrete created by the use of the current methods. The increase of the water mass can be calculated by using the following result:

$$
\Delta m_{w}=m^{*}{ }_{w}-m^{* *}{ }_{w}=21,135-8,023=13,112 \mathrm{~kg}
$$

The increase of the water mass in the concrete production for the parameters of the existing approximate methods in relation to the new proposed exact method is $7,535 \%$. This increase of the water mass negatively affects the quality of the concrete: it reduces the mechanical properties of concrete, increases the porosity and reduces resistance to the low temperature and chemical aggression $[2,3]$. Increasing the water significantly affects the quality of concrete in a negative way, even more than the effect of the aggregate mass reduction. The author believes that this scientific work puts forward sufficient reasons to urgently consider proposals to introduce new methods and new formulas, accurate and iterative, to calculate the wet aggregate and water masses during the dosing into the mixer.

\section{CONCLUSIONS}

The existing methods [4] of calculating the wet aggregate mass according to the Eq. (1) in the process of concrete production are approximate because they neglect the fact that even the corrected, enlarged mass of wet 
aggregate contains water $\left(\Delta m_{a w 2}\right)$, which represents a mistake in the dosing. Because of this error, during the production of concrete, reduced aggregate mass and increased water mass are dosed into the mixture. Depending on the aggregate humidity, the error can adversely affect the concrete quality, because it reduces the mechanical properties and increases the concrete porosity, due to the fact that the mixture obtains the increased water mass. The author provides a new method [1] of calculating the exact aggregate mass $\left(m^{*}{ }_{o a}\right)$ using an iterative process (15) or a direct process (16). The water mass $m^{*}{ }_{w}$ is precisely calculated using the Eq. (17). At the end of the paper, there is a comparative example of calculating the mass of wet aggregates and ground water under the assumption that the humidity of the aggregate is slightly higher $-H_{a}=7,9 \%$. Hence, in a method which is still applied in relation to the proposed new method, we get an error - an increase in the amount of water in the aggregate $\left(\Delta m_{w}=13,112 \mathrm{~kg}\right)$, which represents an increase in the water percentage of $7,535 \%$ relative to the calculated water mass required for this class of concrete. It really is too big of an error that can adversely affect the concrete quality, as we have already pointed out. This method of calculating the exact mass of the liquid and the exact mass of the solid components (mass of the material) according to the presented procedure and the new Eqs. (15), (16), (17), is applicable to all types of mixtures that are applied in the construction, food and pharmaceutical industries.

\section{Acknowledgements}

The work reported in this paper is a part of the investigation within the research project III 42012 "Energy efficiency enhancement of buildings in Serbia and improvement of national regulative capacity for their certification", supported by the Ministry for Science and Technology, Republic of Serbia. This support is gratefully acknowledged.

\section{REFERENCES}

[1] Skenderović, B. \& Kekanović, M. (2011). Građevinski materijali-hemija-struktura-tehnologija-korozija, Textbook Agm knjiga, ISBN 978-86-86363-26-8, Beograd. (in Serbian)

[2] SRPS EN 206-1:2011, Concrete, Serbs Standard, Beograd.

[3] EN 206-1:2000, Concrete, EU Standard, EU.

[4] Kosmatka, S. H., Kerkhoff, B., \& Panarese, W. C. (2003). Design and Control of Concrete Mixtures, EB001, 14 ${ }^{\text {th }}$ edition, Portland Cement Association, Skokie, Illinois, USA.

\section{Contact information:}

Milan KEKANOVIĆ, PhD, Associate Professor

Faculty of Civil Engineering Subotica,

University of Novi Sad,

Kozaračka 2a, 24000 Subotica, Serbia

kekec@gf.uns.ac.rs 